\title{
Intuitionistic fuzzy relations compatible with the group $Z_{n}$
}

\author{
E. G. Emam
}

Correspondence: eg_emom@yahoo. com

Department of Mathematics, Faculty of Science, Zagazig University, Zagazig, Egypt

\begin{abstract}
In this paper, we define the compatibility of finite intuitionistic fuzzy relations with the group $Z_{n}$ and prove some of their fundamental properties. We show that some compositions of $Z_{n}$-compatible intuitionistic fuzzy relations are also $Z_{n}$-compatible intuitionistic fuzzy relation. Also, from any given finite intuitionistic fuzzy relation $\rho$, we can construct two intuitionistic fuzzy relations denoted by $\rho_{L}$ and $\rho_{U}$ which are compatible with $Z_{n}$. We have also provided some examples to clarify the notions and results.
\end{abstract}

Keywords: Intuitionistic fuzzy relations, Fuzzy relations, Compatible fuzzy relations 2010 Mathematics Subject Classification: 15B15, 15B33

\section{Introduction}

In 1965, Zadeh [1] came out with the concept of fuzzy subsets (or fuzzy sets for briefly) which is, indeed, an extension of the classical notion of the ordinary sets. This concept was defined by Zadeh as a function $A: X \rightarrow[0,1]$ where $X$ is a non-empty set. Here, $A$ is a fuzzy subset of $X$ and the number $A(x) \in[0,1]$ is called the degree of membership of the element $x$ in $A$ for every $x \in X$. Zadeh also in [2] defined the fuzzy relation as a fuzzy subset of the Cartesian product $X \times Y$. Subsequently, many researchers [3-7] and others studied fuzzy relations in various contexts. Fuzzy sets and fuzzy relations have many applications in diverse types of sciences, for example, in data bases, pattern recognition, neural network, fuzzy modelling, economy, medicine, and multicriteria decision-making. The concept of Zadeh has, however, some limitations in dealing with uncertainties. Atanasov [8] developed the theory of intuitionistic fuzzy sets as a generalization of fuzzy sets introduced by Zadeh to overcome these difficulties. Atanasov defined the intuitionistic fuzzy set as a pair of fuzzy sets, namely a membership and non-membership functions, which represent positive and negative aspects of the given information. After that, many researchers applied the notion of intuitionistic fuzzy sets to topology, algebra, and other branches of mathematics. Intuitionistic fuzzy set theory is widely applied in solving real-life problems. An example of such application is the optimization in intuitionistic fuzzy environment where by applying this concept, it is possible to reformulate the optimization problem by using degrees of rejection of constraints and values of the objective which are non-admissible. This concept allows one to define the degree of rejection which cannot be simply a complement of the degree of acceptance. The idea of a positive and negative information was

(c) The Author(s). 2019 Open Access This article is distributed under the terms of the Creative Commons Attribution 4.0 International License (http://creativecommons.org/licenses/by/4.0/), which permits unrestricted use, distribution, and reproduction in any medium, provided you give appropriate credit to the original author(s) and the source, provide a link to the Creative Commons license, and indicate if changes were made. 
confirmed by psychological investigations and is widely studied in diverse domains of engineering.

In this paper, we introduce the concept of compatibility of intuitionistic fuzzy relation with the well-known group $\left(Z_{n}, \oplus\right)$ where $\oplus$ is the sum modulo $n$. However, we obtain a characterizations of intuitionistic fuzzy relation compatible with the group $\left(Z_{n}, \oplus\right)$ throughout the isomorphic group $\left(I_{n}, *\right)$ where $I_{n}=\{1,2, \ldots, n\}$.

The paper is organized in four sections. The first section is devoted to the introduction of the paper and the second section is devoted to introduce some definitions and some operations on the ordinary fuzzy relations and intuitionistic fuzzy relations. In the third section, which is the main in this paper, some important results are obtained using the operations and notations introduced in Section 2. The last section is devoted to the conclusion.

\section{Basic Definitions}

We give here some definitions and notations which are applied throughout the paper.

Definition 2.1. [1] Let $X$ be a non-empty set. A function A from $X$ to the unit interval $[0,1]$ is called a fuzzy subset of.X. The number $A(x)$ is called a membership grade of $x$ in A for every $x \in X$

Definition 2.2. [2] Let $X$ be a non-empty set. A function $R$ from $X \times X$ to the unit interval $[0,1]$ is called a fuzzy relation on $X$.

If card $X=n$ where $n \in \mathbb{N}$, then $R$ may be represented by a matrix $\in[0,1]^{n \times n}$ (called a fuzzy matrix). There is a large number of papers on fuzzy matrices from 1977 to now.

Definition 2.3. [8] Let $X$ be a non-empty set and $A, A^{d}: X \rightarrow[0,1]$ be two fuzzy subsets of $X$ such that $A(x)+A^{d}(x) \leq 1$ for every $x \in X$. A pair $\mathcal{F}=\left\langle A, A^{d}\right\rangle$ is called an intuitionistic fuzzy subset of $X$ where $A(x)$ denotes the degree of membership and $A^{d}(x)$ denotes the degree of non-membership of every $x \in X$ to $\mathcal{F}$ respectively.

Definition 2.4. [4] Let $X$ be a nonempty set and $R, R^{d}: X \times X \rightarrow[0,1]$ be two fuzzy relations on $X$ such that $R(x, y)+R^{d}(x, y) \leq 1$ for every $x, y \in X$. A pair $\rho=\left\langle R, R^{d}\right\rangle$ is called an intuitionistic fuzzy relation on $X$.

Similarly, as of ordinary fuzzy relations, if card $X=n$ where $n \in \mathbb{N}$, then $\rho$ may be represented by a matrix $\in([0,1] \times[0,1])^{n \times n}$ (called an intuitionistic fuzzy matrix). Note that the elements of such matrices are taken from the set

$$
F=\left\{\left\langle a, a^{d}\right\rangle: a+a^{d} \leq 1\right\} \text { such that } a, a^{d} \in[0,1]
$$

The family of all intuitionistic fuzzy relations on a non-empty set $X$ is denoted by $\operatorname{IFR}(X)$. If $\rho=\left\langle R, R^{d}\right\rangle$ is an intuitionistic fuzzy relation with $R(x, y)+R^{d}(x, y) \leq 1$ for every $x, y \in X$, then $\rho$ is reduced to be ordinary fuzzy relation. This explains why the concept of intuitionistic fuzzy relations is an extension of that of the ordinary fuzzy ones. If the fuzzy relation $\pi_{\rho}: X \times X \rightarrow[0,1]$ is associated to each intuitionistic fuzzy relation $\rho=\langle R$, $\left.R^{d}\right\rangle$, where $\pi_{\rho}(x, y)=1-R(x, y)-R^{d}(x, y)$ for every $x, y \in X$, the number $\pi_{\rho}(x, y)$ is called an index of an element $(x, y)$ in the intuitionistic fuzzy relation $\rho$. It is also described as an index (a degree) of hesitation whether $x$ and $y$ are in the relation $\rho$ or not. This value is also regarded as a measure of non-determinacy or uncertainty (see [11]), and it is useful in some applications namely in decision-making problems. 
From Definition 2. 4, we see that an intuitionistic fuzzy relation is a pair of fuzzy relations which represent a membership and non-membership functions, respectively. This description is connected with the existence of positive and negative information about the given relation. The symbols $R(x, y)$ and $R^{d}(x, y)$ may be regarded as a lower bound on membership and a lower bound on non-membership of a pair $(x, y)$, respectively.

Some basic operations on intuitionistic fuzzy relations $\operatorname{IFR}(X)$ are extensions of the respective operations on ordinary fuzzy relations. As a result, operations on the set of ordinary fuzzy relations are particular cases of the ones on the set $\operatorname{IFR}(X)$.

Definition 2.5. [3]. Let $\rho=\left\langle R, R^{d}\right\rangle, \sigma=\left\langle S, S^{d}\right\rangle \in \operatorname{IFR}(X)$. Then, the following operations are defined as follows:

$$
\begin{aligned}
& \text { sum : } \rho \vee \sigma=\left\langle R \vee S, R^{d} \wedge S^{d}\right\rangle, \\
& \text { intersection : } \rho \wedge \sigma=\left\langle R \wedge S, R^{d} \vee S^{d}\right\rangle, \\
& \text { composition : } \rho^{\circ} \sigma=\left\langle R \bullet S, R^{d} \circ^{\prime} S^{d}\right\rangle, \\
& \text { complement : } \rho^{c}=\left\langle R^{d}, R\right\rangle
\end{aligned}
$$

where

$$
\begin{aligned}
& (R \vee S)(x, y)=R(x, y) \vee S(x, y)=\max (R(x, y), S(x, y)) x, y \in X \\
& (R \wedge S)(x, y)=R(x, y) \wedge S(x, y)=\min (R(x, y), S(x, y) \quad x, y \in X \\
& (R \circ S)(x, y)=\mathrm{V}_{z \in X}(R(x, z) \wedge S(z, y) \\
& \left(R^{d}{ }^{\prime} S^{d}\right)(x, y)=\bigwedge_{z \in X}\left(R^{d}(x, z) \vee S^{d}(z, y)\right.
\end{aligned}
$$

We may write $\rho^{2}=\rho \circ \rho, \rho^{3}=\rho^{2} \circ \rho$, and in general, $\rho^{k}=\rho^{k-1} \circ \rho$ for every $k \in \mathbb{N}$. If $\rho$, $\sigma \in \operatorname{IFR}(X)$, then we write $\rho \leq \sigma$ if and only if $\rho(x, y) \leq \sigma(x, y)$. That is $\rho \leq \sigma$ if and only if $R(x, y) \leq S(x, y)$ and $R^{d}(x, y) \geq S^{d}(x, y)$ for all $x, y \in X$ and $\rho=\sigma$ if and only if $R(x, y)=S(x$, $y$ ) and $R^{d}(x, y)=S^{d}(x, y)$ for all $x, y \in X$. Also, $\rho<\sigma$ if and only if $R(x, y)<S(x, y)$ and $R^{d}(x, y)>S^{d}(x, y)$ for all $x, y \in X$

Definition 2.6. [4, 12] Let $\rho=\left\langle R, R^{d}\right\rangle \in \operatorname{IFR}(X)$. Then, the converse intuitionistic fuzzy relation of $\rho$ is denoted by $\rho^{-1}$ and is defined as $\rho^{-1}=\left\langle R^{-1},\left(R^{d}\right)^{-1}\right\rangle$, where $R^{-1}(x, y)=R(y$, $x)$ and $\left(R^{d}\right)^{-1}(x, y)=R^{d}(y, x)$ for every $x, y \in X$.

\section{Definition 2.7. $[4,12]$ Let $\rho=\left\langle R, R^{d}\right\rangle \in \operatorname{IFR}(X)$. Then $\rho$ is called an:}

(a) intuitionistic fuzzy symmetric if and only if $\rho^{-1}=\rho$,

(b) intuitionistic fuzzy transitive if and only if $\rho^{2} \leq \rho$, i.e., $\rho(x, y) \wedge \rho(y, z) \leq \rho(x, z)$ for all $x, y, z \in X$,

(c) intuitionistic fuzzy reflexive if and only if $\rho(x, x)=\langle 1,0\rangle$ for all $x \in X$,

(d) intuitionistic fuzzy irreflexive if and only if $\rho(x, x)=\langle 0,1\rangle$ for all $x \in X$.

Definition 2.8. [7, 9, 10] Let $G$ be a multiplicative semi-group. A fuzzy relation $R$ on $G$ is called a left G-compatible fuzzy relation if $R(x a, x b) \geq R(a, b)$ for every $a, b, x \in G$, and it is called a right G-compatible fuzzy relation if $R(a x, b x) \geq R(a, b)$ for every $a, b$, $x \in G$. It is called a G-compatible fuzzy relation if it is left and right G-compatible fuzzy relation. 


\section{$Z_{n}$-Compatible Intuitionistic Fuzzy Relations}

Let $Z_{n}=\left\{l_{1}=\overline{0}, l_{2}=\overline{1}, \ldots, l_{n}=\overline{n-1}\right\}$. Then, as it is well known, $\left(Z_{n}, \oplus\right)$ is an abelian group where the operation $\oplus$ is the sum modulo $n$. Let us put $l_{i} \oplus l_{j}=l_{i * j}$ for every $i$, $j \in I_{n}=\{1,2, \ldots, n\}$ where $n \in \mathbb{N}$. Then, $\left(I_{n}, *\right)$ is also an abelian group where the operation $*$ is defined on the set $I_{n}$ as follows:

$$
i * j=\left\{\begin{array}{c}
i+j-1 \text { if } i+j-1 \leq n \\
i+j-1-n \text { if } i+j-1>n
\end{array}\right.
$$

The mapping $\phi:\left(Z_{n}, \oplus\right) \rightarrow\left(I_{n}, *\right)$ defined by $\phi\left(l_{i} \oplus l_{j}\right)=i * j$ is an isomorphism of groups and the two groups are isomorphic, and so, the intuitionistic fuzzy relations defined on the group $\left(Z_{n}, \oplus\right)$ may be regarded as that of the isomorphic group $\left(I_{n}\right.$, *). In the following, we write $Z_{n}$ instead of $\left(Z_{n}, \oplus\right)$ for simplicity.

Definition 3.1. The relation $\rho=\left\langle R, R^{d}\right\rangle \in \operatorname{IFR}\left(I_{n}, *\right)$ is called a left $Z_{n}$-compatible if and only if $R(i, j) \leq R(k * i, k * j)$ and $R^{d}(i, j) \geq R^{d}(k * i, k * j)$ for every $i, j, k \in I_{n}$. Similarly, $\rho$ is called a right $Z_{n}$-compatible if and only if $R(i, j) \leq R(i * k, j * k)$ and $R^{d}(i, j) \geq R^{d}(i * k, j * k)$ for every $i, j, k \in I_{n}$. From this definition, since the operation $*$ is abelian, we see that when the intuitionistic fuzzy relation $\rho$ is a left $Z_{n}$-compatible, it is also a right $Z_{n^{--}}$ compatible and then we call it $Z_{n}$-compatible. Moreover, since, $\left(I_{n}, *\right)$ is a group and $\rho$ is a $Z_{n}$-compatible, we get

$$
R(i, j) \leq R(i * k, j * k)=R\left(i * k * k^{-1}, j * k * k^{-1}\right)=R(i, j)
$$

and

$$
R^{d}(i, j) \geq R^{d}(i * k, j * k)=R^{d}\left(i * k * k^{-1}, j * k * k^{-1}\right)=R^{d}(i, j)
$$

Thus, an intuitionistic fuzzy relation $\rho=\left\langle R, R^{d}\right\rangle$ is called a $Z_{n}$-compatible if and only if $R(i, j)=R(i * k, j * k)$ and $R^{d}(i, j)=R^{d}(i * k, j * k)$ for every $i, j, k \in I_{n}$, so that $R(i, i)=R(j, j)$ and $R^{d}(i, i)=R^{d}(j, j)$ for every $i, j, k \in I_{n}$. i.e., $\rho(i, i)=\rho(j, j)$ for every $i, j, k \in I_{n}$. Here, the two fuzzy relations $R$ and $R^{d}$ are also $Z_{n}$-compatible.

Remark 3.1. When the two fuzzy relations $R$ and $R^{d}$ in Definition 3.1 are presented by fuzzy matrices, then these fuzzy matrices are completely determined by their first rows (or first column) and so we may write $b_{i}$ to express $\rho(1, i)=\left\langle R(1, i), R^{d}(1, i)\right\rangle$.

Proposition 3.1. Let $\rho=\left\langle R, R^{d}\right\rangle \in \operatorname{IFR}\left(I_{n}, *\right)$. Then, $\rho$ is a $Z_{n}$-compatible if and only if $\rho(i, j)=\rho(i \oplus k, j \oplus k)$ for every $i, j, k \in I_{n}$.

Proof. First, we notice that $i \oplus k=i * k * 2$ for every $i, j, k \in I_{n}$. Now, let $\rho$ be an intuitionistic fuzzy relation compatible with the group $Z_{n}$ and let $i, j, k \in I_{n}$. Then, by the commutativity of $*$ and noting that $k * 2 \in I_{n}$, we have:

$$
\rho(i, j)=\rho(i *(k * 2), j *(k * 2)=\rho(i \oplus k, j \oplus k) .
$$

Conversely, suppose that $\rho(i, j)=\rho(i \oplus k, j \oplus k)$ for every, $j, k \in I_{n}$. Since $k * n \in I_{n}$ and $k * 2=1$, we have:

$$
\begin{aligned}
& \rho(i, j)=\rho(i \oplus(k * n), j \oplus(k * n)) \\
& =\rho(i *(k * n) * 2, j *(k * n) * 2)=\rho(i * k, j * k) .
\end{aligned}
$$

Therefore, $\rho$ is a $Z_{n}$-compatible intuitionistic fuzzy relation. 


\section{Proposition 3.2. The following statements are equivalent:}

(1) $\rho$ is a $Z_{n}$ - compatible intuitionistic fuzzy relation,

(2) $\rho(i, j)=b_{i^{-1} * j}$,

(3) if $\rho(i, j)=b_{k}$, then $\rho(j, i)=b_{k^{-1}}$ for every $i, j, k \in I_{n}$.

Proof. (1) implies (2).

Let $\rho$ be a $Z_{n}$-compatible intuitionistic fuzzy relation. Then

$\rho(i, j)=\rho(i * k, j * k)$ for every $i, j, k \in I_{n}$

and so

$$
\rho(i, j)=\rho\left(i * i^{-1}, j * j^{-1}\right)=\rho\left(1, j * i^{-1}\right)=b_{j * i^{-1}}=b_{i^{-1} * j} .
$$

(2) implies (1).

Let $\rho(i, j)=b_{i^{-1} * j}$ for every $i, j, k \in I_{n}$.

Then,

$$
\rho(i * k, j * k)=b_{(i * k)^{-1} *(j * k)}=b_{k^{-1} * i^{-1} * j * k}=b_{i^{-1} * j}=\rho(i, j) .
$$

Thus, $\rho$ is a $Z_{n}$-compatible intuitionistic fuzzy relation.

(2) implies (3).

Let $\rho(i, j)=b_{i^{-1} * j}$ and let $\rho(i, j)=b_{k}$ for every $i, j, k \in I_{n}$. Then,

$$
\rho(i, j)=b_{k}=b_{i^{-1} * j}
$$

and so

$$
b_{k^{-1}}=b_{\left(i^{-1} * j\right)^{-1}}=b_{j^{-1} * i}=\rho(j, i) .
$$

(3) implies (2).

Suppose that $\rho(j, i)=b_{k^{-1}}$ whenever $\rho(i, j)=b_{k}$ for every $i, j, k \in I_{n}$. We will show that $\rho(i, j)=b_{i^{-1} * j}$.

Since $b_{1^{-1} * i}=b_{i}=\rho(1, i)$, we get

$$
\rho(i, 1)=b_{i^{-1}}=b_{i^{-1} * 1},
$$

Thus,

Therefore,

$$
\rho(1, i)=b_{1^{-1} * i} \text { and } \rho(i, 1)=b_{i^{-1} * 1} .
$$

$\rho(i, j)=b_{i^{-1} * j}$

Remarks 3.2. (1) It follows from Proposition 3.2 that the number of elements of a $Z_{n^{-}}$ compatible intuitionistic fuzzy relation is at most equal $n$.

(2) If a relation $\rho$ is presented by an intuitionistic fuzzy matrix, then we can obtain this matrix from the table of $\left(Z_{n}, \oplus\right)$ by replacing the $i$ th row by $i^{-1}$ th row and $l$ by $b$.

(3) By Proposition 3.1, we can see that:

(i) $\rho(i, j)=b_{(n+j-i+1)}$ with $b_{n+l}=b_{l}$,

(ii) $\rho(i, j)=\rho(i \oplus k, j \oplus k)=\rho(i+1, j+1)$ with $\rho(n+l, n+h)=\rho(1, h)$ for every $i, j, k \in I_{n}$,

(iii) $\rho(n, j)=b_{j+1)}$ for every $j \in I_{n}$.

(4) The rows parallel to the diagonal have the same elements.

Example 3.1. If $=4$, then $Z_{4}=\left\{l_{1}=\overline{0}, l_{2}=\overline{1}, l_{3}=\overline{2}, l_{4}=\overline{3}\right\}$ and the table of $\left(Z_{4}, \oplus\right)$ is as follows: 


$\begin{array}{lllll}\oplus & l_{1} & l_{2} & l_{3} & l_{4} \\ l_{1} & l_{1} & l_{2} & l_{3} & l_{4} \\ l_{2} & l_{2} & l_{3} & l_{4} & l_{1} \\ l_{3} & l_{3} & l_{4} & l_{1} & l_{2} \\ l_{4} & l_{4} & l_{1} & l_{2} & l_{3}\end{array}$

Since $l_{1}^{-1}=l_{1}, l_{2}^{-1}=l_{4}$, and $l_{3}^{-1}=l_{3}$, it follows from Proposition 3.1 that the following matrix:

$$
\rho=\left[\begin{array}{llll}
b_{1} & b_{2} & b_{3} & b_{4} \\
b_{4} & b_{1} & b_{2} & b_{3} \\
b_{3} & b_{4} & b_{1} & b_{2} \\
b_{2} & b_{3} & b_{4} & b_{1}
\end{array}\right]
$$

represents an intuitionistic fuzzy relation compatible with the group $Z_{4}$ where $b_{i} \in F$ for all $i=1,2,3,4$. This matrix is called circulant as it is well known.

Proposition 3.3. Let $\rho=\left\langle R, R^{d}\right\rangle$ and $\sigma=\left\langle S, S^{d}\right\rangle$ be two $Z_{n}$-compatible intuitionistic fuzzy relations. Then, $\rho^{-1}, \rho, \rho \vee \sigma, \rho \wedge \sigma$ and $\rho \circ \sigma$ are also intuitionistic fuzzy relations.

Proof. We only prove that the composition of $\rho$ and $\sigma$ is also a $Z_{n}$-compatible intuitionistic fuzzy relation and the proof of the others are straightforward. Now,

$$
\rho^{\circ} \sigma(i, j)=\left\langle\bigvee _ { l = 1 } ^ { n } \left( R(i, l) \wedge S(l, j), \bigwedge_{l=1}^{n}\left(R^{d}(i, l) \vee S^{d}(l, j)\right\rangle\right.\right.
$$

Since we have that $R, S, R^{d}$ and $S^{d}$ are all $Z_{n}$-compatible fuzzy relations, we write

$$
\begin{aligned}
& \rho \circ \sigma(i, j)=\left\langle\bigvee _ { l = 1 } ^ { n } \left( R(i * k, l * k) \wedge S(l * k, j * k), \bigwedge_{l=1}^{n}\left(R^{d}(i * k, l * k) \vee S^{d}(l * k, j * k)\right\rangle\right.\right. \\
& =\left\langle\bigvee _ { h = 1 } ^ { n } \left( R(i * k, h) \wedge S(h, j * k), \bigwedge_{h=1}^{n}\left(R^{d}(i * k, h) \vee S^{d}(h, j * k)\right\rangle\right.\right.
\end{aligned}
$$

(since $\left(I_{n}, *\right)$ is a group)

$$
=\rho \circ \sigma(i * k, j * k) \text {. }
$$

Therefore, $\rho \circ \sigma$ is a $Z_{n}$-compatible intuitionistic fuzzy relation.

Remarks 3.3. By Proposition 3.4., we have:

(1) If $\rho$ is a $Z_{n}$-compatible intuitionistic fuzzy relation, then so also $\rho^{k}$ for all $k \in \mathbb{N}$.

(2) If $\beta$ is the set of all $Z_{n}$-compatible intuitionistic fuzzy relations, then $(\beta, \wedge),(\beta, \vee)$, and $(\beta, \circ)$ are monoids with unite element $\varepsilon_{1}, \varepsilon_{2}$, and $\varepsilon_{3}$ respectively, where $\varepsilon_{1}, \varepsilon_{2}$, and $\varepsilon_{3}$ and are defined as $\varepsilon_{1}(i, j)=\langle 1, o\rangle, \quad \varepsilon_{2}(i, j)=\langle 0,1\rangle$, and

$$
\varepsilon_{3}(i, j)=\left\{\begin{array}{c}
\langle 1,0\rangle \text { if } i=j \\
\langle 0,1\rangle \text { if } i \neq j
\end{array}\right.
$$

for every $i, j \in I_{n}$.

Definition 3.2. Let $\rho=\left\langle R, R^{d}\right\rangle, \sigma=\left\langle S, S^{d}\right\rangle \in \operatorname{IFR}\left(I_{n}\right)$. Then, we define $\rho \ominus \sigma, \rho \triangleright \sigma \in$ $\operatorname{IFR}\left(I_{n}\right)$ as 


$$
(\rho \ominus \sigma)(i, j)=\left\{\begin{array}{c}
\langle 0,1\rangle \text { if } R(i, j) \leq S(i, j) \text { and } R^{d}(i, j) \geq S^{d}(i, j), \\
\left\langle 0, R^{d}(i, j)\right\rangle \text { if } R(i, j) \leq S(i, j) \text { and } R^{d}(i, j)<S^{d}(i, j), \\
\left\langle R(i, j), R^{d}(i, j)\right\rangle \text { if } R(i, j)>S(i, j) .
\end{array}\right.
$$

and

$$
(\rho \triangleright \sigma)(i, j)=\bigwedge_{k=1}^{n}\left(\left\langle R(i, l), R^{d}(i, l)\right\rangle \cdot \triangleright\left\langle S(l, j), S^{d}(l, j)\right\rangle\right.
$$

where

$$
\begin{aligned}
& \left\langle R(i, j), R^{d}(i, j)\right\rangle \triangleright\left\langle S(l, k) \cdot S^{d}(l, k)\right\rangle \\
= & \left\{\begin{array}{c}
\langle 1,0\rangle \text { if } R(i, j) \leq S(l, k), \\
\langle S(l, k), 0\rangle \text { if } R(i, j)>S(l, k) \text { and } R^{d}(i, j) \geq S^{d}(l, k), \\
\left\langle S(l, k), S^{d}(l, k)\right\rangle \text { if } \mathrm{R}(\mathrm{i}, \mathrm{j})>\mathrm{S}(1, \mathrm{k}) \text { and } R^{d}(i, j)<S^{d}(l, k)
\end{array}\right.
\end{aligned}
$$

for every $i, j, k \in I_{n}$

Proposition 3.4. Let $\rho=\left\langle R, R^{d}\right\rangle$ and $\sigma=\left\langle S, S^{d}\right\rangle$ be two $Z_{n}$-compatible intuitionistic fuzzy relations. Then, $\rho \ominus \sigma$ and $\rho \triangleright \sigma$ are also $Z_{n}$-compatible intuitionistic fuzzy relations.

Proof. To prove that $\rho \ominus \sigma$ is a $Z_{n}$-compatible intuitionistic fuzzy relation, let $\tau=\rho$ $\ominus \sigma$. Then,

$$
\begin{aligned}
& \tau(i, j)=\left\langle T(i, j), T^{d}(i, j)\right\rangle \\
& \quad=\left\{\begin{array}{c}
\langle 0,1\rangle \text { if } \mathrm{R}(\mathrm{i}, \mathrm{j}) \leq \mathrm{S}(\mathrm{i}, \mathrm{j}) \text { and } R^{d}(i, j) \geq S^{d}(i, j) \\
\left\langle 0 . R^{d}(i, j)\right\rangle \text { if } R(i, j) \leq S(i, j) \text { and } R^{d}(i, j)<S^{d}(i, j), \\
\left\langle R(i, j), R^{d}(i, j)\right\rangle \text { if } R(i, j)>S(i, j) .
\end{array}\right.
\end{aligned}
$$

Now, we have three cases:

Case $(i)$. If $R(i, j) \leq S(i, j)$ and $R^{d}(i, j) \geq S^{d}(i, j)$, then

$$
R(i * k, j * k) \leq S(i * k, j * k)
$$

and

$$
R^{d}(i * k, j * k) \geq S^{d}(i * k, j * k) .
$$

Therefore,

$\tau(i, j)=\langle 0,1\rangle=\tau(i * k, j * k)$.

Case (ii). If $R(i, j) \leq S(i, j)$ and $R^{d}(i, j)<S^{d}(i, j)$, then

$$
R(i * k, j * k) \leq S(i * k, j * k)
$$

and

$$
R^{d}(i * k, j * k)<S^{d}(i * k, j * k) .
$$

Therefore,

$$
\tau(i, j)=\left\langle 0, R^{d}(i, j)\right\rangle=\left\langle 0, R^{d}(i * k, j * k)\right\rangle=\tau(i * k, j * k)
$$

Case $(i i i)$. If $R(i, j)>S(i, j)$, then $R(i * k, j * k)>S(i * k, j * k)$ and so

$$
\tau(i * k, j * k)=\left\langle R(i * k, j * k), R^{d}(i * k, j * k)\right\rangle
$$




$$
=\left\langle R(i, j), R^{d}(i, j)\right\rangle=\tau(i, j) .
$$

Therefore, $\rho \ominus \sigma$ is a $Z_{n}$-compatible intuitionistic fuzzy relation.

To show that $\rho \triangleright \sigma$ is a $Z_{n}$-compatible intuitionistic fuzzy relation, let $\eta=\rho \triangleright \sigma$. Then, we have

$$
\begin{aligned}
& \eta(i, j)=\bigwedge_{l=1}^{n}\left(\left\langle R(i, l), R^{d}(i, l)\right\rangle \triangleright\left\langle S(l, j), S^{d}(l, j)\right\rangle\right) \eta(i, j) \\
& =\bigwedge_{l=1}^{n}\left(\left\langle R(i * k, l * k), R^{d}(i * k, l * k)\right\rangle \triangleright\left\langle S(l * k, j * k), S^{d}(l * k, j * k)\right\rangle\right) \\
& =\bigwedge_{h=1}^{n}\left(\left\langle R(i * k, h), R^{d}(i * k, h)\right\rangle \triangleright\left\langle S(h, j * k), S^{d}(h, j * k)\right\rangle\right)
\end{aligned}
$$

(since $\left(I_{n}, *\right)$ is a group)

$=\eta(i * k, j * k)$ for every $i, j, k \in I_{n} \cdot \rho$.

Thus, the intuitionistic fuzzy relation $\rho \triangleright \sigma$ is a $Z_{n}$-compatible.

Corollary 3.1. Let $\rho$ be a $Z_{n}$-compatible intuitionistic fuzzy relation. Then, $\nabla \rho$ and $\Delta \rho$ are $Z_{n}$-intuitionistic fuzzy relations where $\nabla \rho=\rho \wedge \rho^{-1}$ and $\Delta \rho=\rho \ominus \rho^{-1}$.

Proof. By Propositions 3.3 and 3.4.

It is noted that if $\rho \in \operatorname{IFR}(X)$ for any non-empty set $X$, then $\nabla \rho$ is an intuitionistic fuzzy symmetric and $\Delta \rho$ is an intuitionistic fuzzy irreflexive. Moreover, $\rho=\Delta \rho \vee \nabla \rho$ (see [4])

Example 3.2. Let $n=3$ and $\rho=\left\langle R, R^{d}\right\rangle, \sigma=\left\langle S, S^{d}\right\rangle \in \operatorname{IFR}\left(I_{3}\right)$ be presented by matrices:

$$
R=\left[\begin{array}{lll}
0.5 & 0.6 & 0.7 \\
0.7 & 0.5 & 0.6 \\
0.6 & 0.7 & 0.5
\end{array}\right], R^{d}=\left[\begin{array}{lll}
0.4 & 0.2 & 0.3 \\
0.3 & 0.4 & 0.2 \\
0.2 & 0.3 & 0.4
\end{array}\right]
$$

and

$$
S=\left[\begin{array}{lll}
0.6 & 0.7 & 0.5 \\
0.5 & 0.6 & 0.7 \\
0.7 & 0.5 & 0.6
\end{array}\right], S^{d}=\left[\begin{array}{lll}
0.3 & 0.3 & 0.4 \\
0.4 & 0.3 & 0.3 \\
0.3 & 0.4 & 0.3
\end{array}\right]
$$

Then, it is easy to see that both $\rho$ and $\sigma$ are $Z_{3}$-compatible and

$$
\begin{aligned}
& \rho \ominus \sigma=\left[\begin{array}{ccc}
\langle 0,1\rangle & \langle 0,0.2\rangle & \langle 0.7,0.3\rangle \\
\langle 0.7,0.3\rangle & \langle 0,1\rangle & \langle 0,0.2\rangle \\
\langle 0,0.2\rangle & \langle 0.7,0.3\rangle & \langle 0,1\rangle
\end{array}\right], \\
& \rho \triangleright \sigma=\left[\begin{array}{ccc}
\langle 0.5,0.4\rangle & \langle 0.5,0.4\rangle & \langle 0.6,0\rangle \\
\langle 0.6,0\rangle & \langle 0.5,0.4\rangle & \langle 0.5,0.4\rangle \\
\langle 0.5,0.4\rangle & \langle 0.6,0\rangle & \langle 0.5,0.4\rangle
\end{array}\right]
\end{aligned}
$$

which they are also $Z_{3}$-compatible intuitionistic fuzzy relations.

Let $\varepsilon=\left\langle E, E^{d}\right\rangle \in \operatorname{IFR}\left(I_{n}\right.$, *) be presented by matrices:

$$
E=\left[\begin{array}{ccc}
0 & \cdots & 1 \\
\vdots & \ddots & \vdots \\
1 & \cdots & 0
\end{array}\right] \text { and } E^{d}=\left[\begin{array}{ccc}
1 & \cdots & 0 \\
\vdots & \ddots & \vdots \\
0 & \cdots & 1
\end{array}\right]
$$

Thus, 


$$
\varepsilon=\left[\begin{array}{ccc}
\langle 0,1\rangle & \cdots & \langle 1,0\rangle \\
\vdots & \ddots & \vdots \\
\langle 1,0\rangle & \cdots & \langle 0,1\rangle
\end{array}\right]
$$

i.e.,

$$
\varepsilon(i, j)=\left\{\begin{array}{l}
\langle 1,0\rangle \text { if } i+j=n+1, \\
\langle 0,1\rangle \text { otherwise. }
\end{array} .\right.
$$

Then, we have the following proposition.

Proposition 3.5. Let $\rho \in \operatorname{IFR}\left(I_{n}\right.$, *) be a $Z_{n}$-compatible. Then, $\varepsilon \circ \rho$ is an intuitionistic fuzzy symmetric.

Proof. Let $\tau=\varepsilon \circ \rho$. Then, from the definition of the intuitionistic fuzzy relation $\varepsilon$, we can see that $\tau(i, j)=\rho(n-i+1, j)$ for every $i, j \in I_{n}$. Since we have that $\rho$ is a $Z_{n}$-compatible, we get for every $i, j \in I_{n}$

$\tau(i, j)=\rho(n-i+1 \oplus k, j \oplus k)$ (by Proposition 3.2)

and so

$$
\tau(i, j)=\rho(n-i+1 \oplus k, j \oplus k)=\rho(1, j \oplus i)=b_{j \oplus i} .
$$

Similarly, we can prove that $\tau(i, j)=b_{j \oplus i}$. Hence, $\tau$ is intuitionistic fuzzy symmetric.

Proposition 3.6. Let $\rho \in \operatorname{IFR}\left(I_{n}\right.$, *) be a $Z_{n}$-compatible. Then, $\rho$ is an intuitionistic fuzzy symmetric if and only if $\rho(1, i)=\rho(1, n+2-i)$ for every $i \in I_{n}$

Proof. Let $\rho$ be intuitionistic fuzzy symmetric. Then,

$\rho(1, i)=\rho(k, i * k)=\rho(i * k, k)$ for every $k \in I_{n}$.

Taking $k=n-i$, we get

$$
\rho(1, i)=\rho(n-1, n-i)=\rho(n, n-i+1)=\rho(1, n-i+2) .
$$

Conversely, suppose $\rho(1, i)=\rho(1, n-i+2)$ for every $i \in I_{n}$. Then, $\rho(i, 1)=\rho(i * k, k)$ for every $k \in I_{n}$. Taking also $k=n-i$, we get $\rho(i, 1)=\rho(1, n-i+2)$. Thus, $\rho(1, i)=\rho(i, 1)$.

Since we have that $\rho$ is a $Z_{n}$-compatible, we conclude

$$
\rho(1, i)=\rho(2, i+1)=\rho(3, i+2)=\ldots=\rho(n, i+n-1) .
$$

and

$$
\rho(i, 1)=\rho(i+1,2)=\rho(i+2,3)=\ldots=\rho(i+n-1, n) .
$$

But $\rho(1, i)=\rho(i, 1)$, so that $\rho(u, v)=\rho(v, u)$ for every $u, v \in I_{n}$. Therefore, $\rho$ is intuitionistic fuzzy symmetric. This completes the proof.

Corollary 3.2. Let $\rho \in \operatorname{IFR}\left(I_{n}, *\right)$ be a $Z_{n}$-compatible. Then $\rho$ is an intuitionistic fuzzy symmetric. If and only if $\rho(1, i)=\rho\left(1, i^{-1}\right)$ for every $i \in I_{n}$.

Proof. Since $i^{-1}=n-i+2$ (for $i \neq 1$ ) with respect to the operation * defined on the group $\left(I_{n}, *\right)$.

\section{Note:}

Using Proposition 3.6, the intuitionistic fuzzy relation in Example 3.1 is intuitionistic fuzzy symmetric if and only if $b_{2}=b_{4}$ since $2^{-1}=4$ and $3^{-1}=3$.

Theorem 3.1. Let $\rho=\left\langle R, R^{d}\right\rangle, \sigma=\left\langle S, S^{d}\right\rangle \in \operatorname{IFR}\left(I_{n}\right)$ be two $Z_{n}$-compatible. Then, $\rho$ $\circ \sigma=\sigma \circ \rho$. 
Proof. Let $\tau=\left\langle T, T^{d}\right\rangle=\rho \circ \sigma$ and $\eta=\left\langle Q, Q^{d}\right\rangle=\sigma \circ \rho$. Then, $\tau$ and $\eta$ are $Z_{n}$-compatible by Proposition 3.3. Now, $t(1, k)=\left\langle T(1, k), T^{d}(1, k)\right\rangle$

$$
=\left\langle\bigvee_{l=1}^{n}(R(1, l) \wedge S(l, k)), \bigwedge_{l=1}^{n}\left(R^{d}(1, l) \vee S^{d}(l, k)\right\rangle\right.
$$

and

$$
\begin{aligned}
& \eta(1, k)=\left\langle Q(1, k), Q^{d}(1, k)\right\rangle \\
& =\left\langle\bigvee_{l=1}^{n}(S(1, l) \wedge R(l, k)), \bigwedge_{l=1}^{n}\left(S^{d}(1, l) \vee R^{d}(l, k)\right\rangle\right.
\end{aligned}
$$

In the following, we show that $T(1, k)=Q(1, k)$ and similarly $\left.T^{d}(1, k)=Q^{d}(1, k)\right)$. Since $\tau$ and $\sigma$ are $Z_{n}$-compatible intuitionistic fuzzy relations, the fuzzy relations $T$ and $Q$ are also $Z_{n}$-compatible. We rewrite $T(1, k)$ as follows:

$$
\begin{aligned}
& T(1, k)=\bigvee_{l=1}^{n}(R(1, l) \wedge S(l, k)= \\
& =\bigvee_{l=1}^{k}(R(1, l) \wedge S(1, k+1-l)) \bigvee_{l=k+1}^{n}(R(1, l) \wedge S(l, n+k+1-l)) \\
& =(R(1,1) \wedge S(1, k)) \vee(R(1,2) \wedge S(1, k-1) \\
& \vee(R(1,3) \wedge S(1, k-2)) \vee \ldots \vee(R(1, k) \wedge S(1,1)) \\
& \vee(R(1, k) \wedge S(1,1) \vee(R(1, k-1) \wedge S(1, n)) \\
& \vee(R(1, k+2) \wedge S(1, n-2) \\
& \vee \\
& \vee(R(1, n-1) \wedge S(1, k+2)) \\
& \vee(R(1, n) \wedge S(1, k+1)
\end{aligned}
$$

Similarly, we compute $Q(1, k)$ as follows:

$$
\begin{aligned}
& Q(1, k)=\bigvee_{l=1}^{n}(S(1, l) \wedge R(l, k) \\
& =\bigvee_{l=1}^{k}(S(1, l) \wedge R(1, k+1-l)) \bigvee_{l=k+1}^{n}(S(1, l) \wedge R(l, n+k+1-l)) \\
& =(S(1,1) \wedge R(1, k)) \vee(S(1,2) \wedge R(1, k-1) \\
& \vee(S(1,3) \wedge R(1, k-2)) \vee \ldots \vee(S(1, k) \wedge R(1,1)) \\
& \vee(S(1, k) \wedge R(1,1) \vee(S(1, k-1) \wedge R(1, n)) \\
& \vee(S(1, k+2) \wedge R(1, n-2)) \\
& \vee \\
& \vee(S(1, n-1) \wedge R(1, k+2)) \\
& \vee(S(1, n) \wedge R(1, k+1)
\end{aligned}
$$

From these computations of $T(1, k)$ and $Q(1, k)$, it is clear that $=Q(1, k)$. In a similar way, we can show that $T^{d}(1, k)=Q^{d}(1, k)$. Thus, $\tau(1, k)=\eta(1, k)$. Again, since $\tau$ and $\eta$ are $Z_{n}$-compatible intuitionistic fuzzy relations and $\tau(1, k)=\eta(1, k)$, we get $\tau=\eta$, i.e., $\rho$ $\circ \sigma=\sigma \circ \rho$.

Definition 3.2. For $\rho=\left\langle R, R^{d}\right\rangle \in I F R\left(I_{n}\right)$, we define the two intuitionistic fuzzy relations $\rho_{L}=\left\langle R_{L},\left(R^{d}\right)_{L}\right\rangle$ and $\rho_{U}=\left\langle R_{U},\left(R^{d}\right)_{U}\right\rangle$ as follows:

$$
R_{L}(i, j)=\min \left\{R(u * i * k * v, u * j * k * v), \quad i, j, k, u, v \in I_{n}\right\},
$$




$$
\left(R^{d}\right)_{L}(i, j)=\max \left\{R^{d}(u * i * k * v, u * j * k * v), \quad i, j, k, u, v \in I \_n\right\}
$$

and

$$
\begin{aligned}
& R_{U}(i, j)=\max \left\{R(u * i * k * v, u * j * k * v), \quad i, j, k, u, v \in I_{n}\right\}, \\
& \left(R^{d}\right)_{U}(i, j)=\min \left\{R^{d}(u * i * k * v, u * j * k * v), \quad i, j, k, u, v \in I \_n\right\}
\end{aligned}
$$

The following two propositions have interesting properties. They enable us to construct two $Z_{n}$-compatible intuitionistic fuzzy relations $\rho_{L}$ and $\rho_{U}$ on $\left(I_{n}\right.$ * $)$ from any given intuitionistic fuzzy relation $\rho$ on $\left(I_{n}, *\right)$. Moreover, $\rho_{L} \leq \rho \leq \rho_{U}$.

Proposition 3.7. Let $\rho=\left\langle R, R^{d}\right\rangle \in \operatorname{IFR}\left(I_{n}\right)$. Then, $\rho_{L} \in \operatorname{IFR}\left(I_{n}\right.$ * is a $Z_{n}$-compatible intuitionistic fuzzy relation. Moreover, it is the largest one contained in $\rho$.

Proof. First, we notice that the number $u * k * v$ must be one of the different elements of the group $\left(I_{n}, *\right)$ and so also $u * v$. Now, we show that the intuitionistic fuzzy relation $\rho_{L}$ is a $Z_{n}$-compatible. We have:

$$
\begin{aligned}
& R_{L}(i, j)=\min \left\{R(u * i * v, u * j * v), \quad i, j, u, v \in I_{n}\right\} \\
& \quad=\min \{R(i * 1, j * 1),(R(i * 2, j * 2), \ldots,(R(i * n, j * n)\} .
\end{aligned}
$$

Also,

$$
\begin{aligned}
& R_{L}(i * k, j * k)=\min \left\{R(u * i * k * v, u * j * k * v), i, j, k, u, v \in I_{n}\right\} \\
& =\min \{R(i * 1, j * 1), R(i * 2, j * 2), \ldots, R(i * n, j * n)\} .
\end{aligned}
$$

Therefore, $R_{L}(i, j)=R_{L}(i * k, j * k)$. Hence, the fuzzy relation $R_{L}$ is a $Z_{n}$-compatible fuzzy relation. Similarly, we can prove that the fuzzy relation $\left(R^{d}\right)_{L}$ is a $Z_{n}$-compatible fuzzy relation. Therefore, $\rho_{L}$ is a $Z_{n}$-compatible intuitionistic fuzzy relation.

By the definition of $\rho_{L}$ it is clear that $R_{L}(i, j) \leq R(i, j)$ and also, $\left(R^{d}\right)_{L}(i, j) \geq R^{d}(i, j)$ Thus, $\rho_{L} \leq \rho$.

Now, let $\xi=\left\langle L, L^{d}\right\rangle \in \operatorname{IFR}\left(I_{n}, *\right)$ be a $Z_{n}$-compatible such that $\xi \leq \rho$. We will show that $\xi \leq \rho_{L}$. Since we have that

$L(i, j)=L(u * i, u * j)=L(u * i * v, u * j * v) \leq R(u * i * v, u * j * v)$ for every $i, j, u, v \in I_{n}$, we obtain

$$
L(i, j) \leq \min \left\{R(u * i * v, u * j * v): i, j, u, v \in I_{n}\right\}=R_{L}(i, j) .
$$

Similarly, we can show that

$$
L^{d}(i, j) \geq \max \left\{R^{d}(u * i * v, u * j * v): i, j, u, v \in I_{n}\right\}=\left(R^{d}\right)_{L}(i, j) .
$$

Thus, $\xi \leq \rho_{L}$.

Proposition 3.8: Let $\rho=\left\langle R, R^{d}\right\rangle, \rho_{1}=\left\langle R_{1}, R_{1}^{d}\right\rangle, \rho_{2}=\left\langle R_{2}, R_{2}^{d}\right\rangle \in \operatorname{IFR}\left(I_{n}, *\right)$. Then, we have:

(1) $\rho_{1} \leq \rho_{2}$ implies $\left(\rho_{1}\right)_{U} \leq\left(\rho_{2}\right)_{U}$

(2) $\rho_{U}$ is a $Z_{n}$-compatible

(3) $\left(\rho_{U}\right)^{-1}=\left(\rho^{-1}\right)_{U}$

(4) $\left(\rho_{1} \vee \rho_{2}\right)_{U}=\left(\rho_{1}\right)_{U} \mathrm{~V}\left(\rho_{2}\right)_{U}$

Proof. The proof of (1) is clear and the proof of (2) is similar to the proof of $\rho_{L}$ in Proposition 3.7. However, we only prove (3) and (4). 
(3) Let $\rho_{U}=\left\langle R_{U},\left(R^{d}\right)_{U}\right\rangle \in \operatorname{IFR}\left(I_{n}, *\right)$. Then,

$$
\begin{aligned}
& \left(R_{U}\right)^{-1}(i, j)=R_{U}(j, i)=\max \left\{R(u * j * v, u * i * v): i, j, u, v \in I_{n}\right\} \\
& =\max \left\{R^{-1}(u * j * v, u * i * v): i, j, u, v \in I_{n}\right\} \\
& =\left(R^{-1}\right)_{U}
\end{aligned}
$$

Thus,

$$
\left(R_{U}\right)^{-1}=\left(R^{-1}\right)_{U}
$$

Similarly, we can show that $\left(\left(R^{d}\right)_{U}\right)^{-1}=\left(\left(R^{d}\right)^{-1}\right)_{U}$. This completes the proof and hence $\left(\rho_{U}\right)^{-1}=\left(\rho^{-1}\right)_{U}$

(4) $\quad\left(\rho_{1}\right)_{U} \vee\left(\rho_{2}\right)_{U}=\left\langle\left(\left(R_{1}\right)_{U} \vee\left(R_{2}\right)_{U}\right),\left(\left(R_{1}^{d}\right)_{U} \wedge\left(R_{2}^{d}\right)_{U}\right\rangle\right.$, and $\left(\rho_{1} \vee \rho_{2}\right)_{U}=\left\langle\left(R_{1} \vee R_{2}\right)_{U}\right.$, $\left.\left(R_{1}^{d} \wedge R_{2}^{d}\right)_{U}\right\rangle$.

Now, we show that $\left(\left(R_{1}\right)_{U} \vee\left(R_{2}\right)_{U}\right)=\left(R_{1} \vee R_{2}\right)_{U}$. For every $i, j, u, v \in I_{n}$, we have:

$$
\begin{aligned}
& \left(\left(R_{1}\right)_{U} \vee\left(R_{2}\right)_{U}\right)(i, j)=\max \left(\left(R_{1}\right)_{U}(i, j),\left(R_{2}\right)_{U}(i, j)\right) \\
& =\max \left(\max \left\{R_{1}(u * i * v, u * j * v)\right\}, \max \left\{R_{2}(u * i * v, u * j * v)\right\}\right) \\
& =\max \left(\left\{\max \left(R_{1}(u * i * v, u * j * v)\right\},\left\{R_{2}(u * i * v, u * j * v)\right\}\right)\right. \\
& =\max \left\{\left(R_{1} \vee R_{2}\right)(u * i * v, u * j * v\}=\left(R_{1} \vee R_{2}\right)_{U}(i, j) .\right.
\end{aligned}
$$

Also, for every $i, j, u, v \in I_{n}$ we have:

$$
\begin{aligned}
& \left(\left(R_{1}^{d}\right)_{U} \wedge\left(R_{2}^{d}\right)_{U}\right)(i, j)=\min \left(\left(R_{1}^{d}\right)_{U}(i, j),\left(R_{2}^{d}\right)_{U}(i, j)\right. \\
& =\min \left(\operatorname { m i n } \left\{\left(R_{1}^{d}(u * i * k * v, u * j * v\}, \min \left\{\left(R_{2}^{d}(u * i * k * v, u * j * k * v\}\right)\right.\right.\right.\right. \\
& =\min \left(\left\{\operatorname { m i n } \left(R_{1}^{d}(u * i * v, u * j * v\},\left\{\left(R_{2}^{d}(u * i * v, u * j * v\}\right)\right.\right.\right.\right. \\
& =\min \left\{\left(R_{1}^{d} \wedge R_{2}^{d}\right)(u * i * v, u * j * v)\right\}=\left(R_{1}^{d} \wedge R_{2}^{d}\right)_{U}(i, j) .
\end{aligned}
$$

Therefore, $\left(\rho_{1} \vee \rho_{2}\right)_{U}=\left(\rho_{1}\right)_{U} \vee\left(\rho_{2}\right)_{U}$.

Analogously, we can prove the following proposition.

Proposition 3.9. Let $\rho=\left\langle R, R^{d}\right\rangle, \rho_{1}=\left\langle R_{1}, R_{1}^{d}\right\rangle, \rho_{2}=\left\langle R_{2}, R_{2}^{d}\right\rangle \in \operatorname{IFR}\left(I_{n}, *\right)$. Then, we have:

(1) $\rho_{1} \leq \rho_{2}$ implies $\left(\rho_{1}\right)_{L} \leq\left(\rho_{2}\right)_{L}$

(2) $\left(\rho_{L}\right)^{-1}=\left(\rho^{-1}\right)_{L}$

(3) $\left(\rho_{1} \wedge \rho_{2}\right)_{L}=\left(\rho_{1}\right)_{L} \wedge\left(\rho_{2}\right)_{L}$

Corollary 3.3. Let $\rho$ be intuitionistic reflexive (irreflexive). Then, $\rho_{U}\left(\rho_{L}\right)$ is intuitionistic fuzzy reflexive (irreflexive).

Proposition 3.10. Let $\rho=\left\langle R, R^{d}\right\rangle \in \operatorname{IFR}\left(I_{n}\right.$, *). If $\rho$ is a $Z_{n}$-compatible, then $\rho_{L}=\rho_{U}=\rho$.

Proof Let $\rho_{U}=\left\langle R_{U},\left(R^{d}\right)_{U}\right\rangle$ be a $Z_{n}$-compatible intuitionistic fuzzy relation. Then, both $R_{U}$ and $\left(R^{d}\right)_{U}$ are also $Z_{n}$-compatible fuzzy relations and so,

$$
R_{U}(i, j)=\max \left\{\left(R(u * i * v, u * j * v), \quad i, j, u, v \in I_{n}\right\}=R(i, j)\right.
$$




$$
\left(R^{d}\right)_{U}(i, j)=\min \left\{\left(R^{d}(u * i * v, u * j * v), \quad i, j, u, v \in I_{n}\right\}=R(i, j)\right.
$$

Therefore, $\rho_{U}=\rho$.

Similarly, it can be shown that $\rho_{L}=\rho$.

Example 3.3: For $n=4$, let $\rho=\left\langle R, R^{d}\right\rangle \in \operatorname{IFR}\left(I_{4}\right)$ be presented by matrices:

$$
R=\left[\begin{array}{cccc}
0.5 & 0.7 & 0.8 & 0.2 \\
0.8 & 0.3 & 0.4 & 0.9 \\
0.7 & 0.5 & 0.3 & 0.6 \\
0.9 & 1.0 & 0 & 0.4
\end{array}\right] \text { and } R^{d}=\left[\begin{array}{cccc}
0.5 & 0.3 & 0.2 & 0.6 \\
0.1 & 0.6 & 0.6 & 0 \\
0.3 & 0.4 & 0.5 & 0.4 \\
0.1 & 0 & 1.0 & 0.3
\end{array}\right]
$$

Thus,

$$
\rho=\left[\begin{array}{cccc}
\langle 0.5,0.5\rangle & \langle 0.7,0.3\rangle & \langle 0.8,0.2\rangle & \langle 0.2,0.6\rangle \\
\langle 0.8,0.1\rangle & \langle 0.3,0.6\rangle & \langle 0.4,0.6\rangle & \langle 0.9,0\rangle \\
\langle 0.7,0.3\rangle & \langle 0.5,0.4\rangle & \langle 0.3,0.5\rangle & \langle 0.6,0.4\rangle \\
\langle 0.9,0.1\rangle & \langle 1,0\rangle & \langle 0,1\rangle & \langle 0.4,0.3\rangle
\end{array}\right]
$$

Since

$$
\begin{aligned}
& R_{U}=\left[\begin{array}{cccc}
0.5 & 0.9 & 1 & 0.8 \\
0.8 & 0.5 & 0.9 & 1 \\
1 & 0.8 & 0.5 & 0.9 \\
0.9 & 1 & 0.8 & 0.5
\end{array}\right] \text { and }\left(R^{d}\right)_{U}=\left[\begin{array}{cccc}
0.3 & 0.1 & 0.0 & 0.1 \\
0.1 & 0.3 & 0.1 & 0.0 \\
0.0 & 0.1 & 0.3 & 0.1 \\
0.1 & 0.0 & 0.1 & 0.3
\end{array}\right] \\
& R_{L}=\left[\begin{array}{cccc}
0.3 & 0.4 & 0.7 & 0 \\
0 & 0.3 & 0.4 & 0.7 \\
0.7 & 0 & 0.3 & 0.4 \\
0.4 & 0.7 & 0 & 0.3
\end{array}\right] \text { and }\left(R^{d}\right)_{L}=\left[\begin{array}{cccc}
0.6 & 0.6 & 0.3 & 1 \\
1 & 0.6 & 0.6 & 0.3 \\
0.3 & 1 & 0.6 & 0.6 \\
0.6 & 0.3 & 1 & 0.6
\end{array}\right]
\end{aligned}
$$

we have:

$$
\rho_{U}=\left[\begin{array}{cccc}
\langle 0.5,0.3\rangle & \langle 0.9,0.1\rangle & \langle 1,0\rangle & \langle 0.8,0,1\rangle \\
\langle 0.8,0,1\rangle & \langle 0.5,0.3\rangle & \langle 0.9,0.1\rangle & \langle 1,0\rangle \\
\langle 1,0\rangle & \langle 0.8,0,1\rangle & \langle 0.5,0.3\rangle & \langle 0.9,0.1\rangle \\
\langle 0.9,0.1\rangle & \langle 1,0\rangle & \langle 0.8,0,1\rangle & \langle 0.5,0.3\rangle
\end{array}\right]
$$

and

$$
\rho_{L}=\left[\begin{array}{cccc}
\langle 0.3,0.6\rangle & \langle 0.4,0.6\rangle & \langle 0.7,0.3\rangle & \langle 0,1\rangle \\
\langle 0,1\rangle & \langle 0.3,0.6\rangle & \langle 0.4,0.6\rangle & \langle 0.7,0.3\rangle \\
\langle 0.7,0.3\rangle & \langle 0,1\rangle & \langle 0.3,0.6\rangle & \langle 0.4,0.6\rangle \\
\langle 0.4,0.6\rangle & \langle 0.7,0.3\rangle & \langle 0,1\rangle & \langle 0.3,0.6\rangle
\end{array}\right] .
$$

It is clear that the fuzzy relations $R_{U},\left(R^{d}\right)_{U}, R_{L}$, and $\left(R^{d}\right)_{L}$ are all $Z_{4}$-compatible and so $\rho_{U}$ and $\rho_{L}$ are $Z_{4}$-compatible intuitionistic fuzzy relations. Also, we see that $R_{L} \leq R \leq$ $R_{U}$ and $\left(R^{d}\right)_{L} \geq R \geq\left(R^{d}\right)_{U}$. So that $\rho_{L} \leq \rho \leq \rho_{U}$.

As an instance, we compute $R_{U}(2,3)$ and $\left(R^{d}\right)_{U}(2,3)$ as follows:

$R_{U}(2,3)=\max \left\{R(u * 2 * v, u * 3 * v): u, v \in I_{4}\right\}$. Since we have that $\left(I_{4}, *\right)$ is an abelian group, we conclude $u * v$ must be one of the numbers $1,2,3$ and 4 . So that

$$
\begin{aligned}
& R_{U}(2,3)=\max \{R(2 * 1,3 * 1), R(2 * 2,3 * 2), R(2 * 3,3 * 3), R(2 * 4,3 * 4)\} \\
& =\max \{R(2,3), R(3,4), R(4,1), R(1,2)\} \\
& =\max \{0.4,0.6,0.9,0.7\}=0.9
\end{aligned}
$$

Also 


$$
\left(R^{d}\right)_{U}(2,3)=\min \left\{R^{d}(2,3), R^{d}(3,4), R^{d}(4,1), R^{d}(1,2)\right\}=0
$$

Then

$$
\rho_{U}(2,3)=\langle 0.9,0.1\rangle
$$

Similarly, we have $\rho_{L}(2,3)=\langle 0.4,0.6\rangle$

\section{Conclusion}

The operations $\vee, \wedge, *$ and $\oplus$ play an important role in the whole of the paper. We explored the interesting properties of intuitionistic fuzzy relations which are compatible with the group $\left(Z_{n}, \oplus\right)$ which isomorphic to the group $\left(I_{n}, *\right)$. These relations are, of course, finite and they are circulants. Given any finite intuitionistic fuzzy relation $\rho$, we define in Section 3 two finite intuitionistic fuzzy relations $\rho_{L}$ and $\rho_{U}$. We showed that these relations are compatible with the group $\left(Z_{n}, \oplus\right)$, and hence they are circulants. Thus, this result enables us to construct two circulant intuitionistic fuzzy relations from any given one.

\section{Abbreviations}

IFR $(X)$ : The family of intuitionistic fuzzy relations on a nonempty set $X_{i} \operatorname{IFR}\left(I_{n}, *\right)$ : The family of intuitionistic fuzzy relations on the group $\left(I_{n}, *\right)$, where $I_{n}=\{1,2, \ldots, n\}$

\section{Acknowledgements}

The author is grateful to the referees and the editors for their valuable comments and suggestions in revising and improving the manuscript.

\section{Authors' contributions}

I design this work and draft and revised it, and I have approved the submitted version. Also, I ensure that questions related to the accuracy or integrity of any part of the work.

Funding

Not applicable (no funding for this work from any organization).

Availability of data and materials

Not applicable

\section{Competing interests}

"The author declares that he/she has no competing interests.

Received: 27 February 2019 Accepted: 4 November 2019

Published online: 19 December 2019

\section{References}

1. Zadeh, L.: A: Fuzzy sets. Information and Control. 8, 338-353 (1965)

2. Zadeh, L.: A: Similarity relations and fuzzy orderings. Information Science. 3, 177-200 (1971)

3. Chon, I.: Redefined fuzzy congruences on semigroups. Korean Journal of Mathematics. 22, 621-632 (2014)

4. Dudziak, U.: Pekala B: Equivalent bipolar fuzzy relations. Fuzzy Sets and Systems. 161, 234-253 (2010)

5. Hur, K., Jang, S.Y., Kang, H.W.: Some intuitionistic fuzzy congruences. Iranian Journal of Fuzzy Systems. 3(1), 45-57 (2006)

6. Samhan, M.: Fuzzy congruences on semigroups. Information Science. 74, 165-175 (1993)

7. Sidky, F.I., Ghanim, M.H.: Congruence fuzzy relations on semigroups. Simon steven, a Quarterly journal of Pure and Applied Mathematics. 62, 143-152 (1988)

8. Atanasov, K.T.: Intuitionistic fuzzy sets. Fuzzy Sets and Systems. 20, 87-96 (1986)

9. Samhan, M.: Fuzzy congruences on groups and rings. International Journal of Mathematics and Mathematical Sciences. 17, 468-474 (1994)

10. Kondo, M.: Fuzzy congruences on groups. Quasigroups and Related Systems. 11, 59-70 (2004)

11. Lin, L., Yuan, X.-H., Xia, Z.-Q.: Multicriteria fuzzy decision -making methods based on intuitionistic fuzzy sets. Journal of Computer and System Sciences. 73, 84-88 (2007)

12. Emam, E.G., Fendh, M.: F: Some results associated with the max-min and min-max compositions of bifuzzy matrices. Journal of the Egyptian Mathematical Society. 24, 515-521 (2016)

\section{Publisher's Note}

Springer Nature remains neutral with regard to jurisdictional claims in published maps and institutional affiliations. 